COUGH

\title{
Capsaicin cough sensitivity in bronchiectasis
}

A Torrego, R A Haque, L T Nguyen, M Hew, D H Carr, R Wilson, K F Chung

Thorax 2006:61:706-709. doi: 10.1136/thx.2005.049767

Background: Bronchiectasis is a suppurative airway disease characterised by persistent cough and sputum production associated with bronchial dilatation. A study was undertaken to determine whether cough sensitivity is increased in bronchiectatic patients.

See end of article for authors' affiliations Methods: Twenty two patients with bronchiectasis and 20 healthy non-smoking controls matched for age and sex were recruited into the study. Quality of life (Leicester Cough Questionnaire score), total cough symptom score, and extent of bronchiectasis on HRCT scans were recorded. Cough sensitivity was assessed using incremental inhalation of capsaicin concentrations; the concentration at which 5 or more coughs occurred (C5) was recorded.

Correspondence to: Professor K F Chung National Heart and Lung Institute, Imperial College, Dovehouse Street, London SW3 6LY, UK; f.chung@ imperial.ac.uk

Received 19 July 2005 Accepted 15 March 2006 Published Online First 6 April 2006

Results: Patients with bronchiectasis had increased sensitivity to capsaicin compared with controls (mean (SE) $\log _{10}$ C5 $\left.1.22(0.20) \vee 1.89(0.21) ; p<0.03\right)$. Capsaicin sensitivity correlated positively with the Leicester Cough Questionnaire score $(r=0.64 ; p=0.005)$ and inversely with the total cough symptom score $(r=-0.58 ; p=0.004)$, but not with the extent of the disease. It also correlated with forced expiratory volume in 1 second $\left(\mathrm{FEV}_{1}\right)$ in litres $(r=0.58 ; \mathrm{p}=0.005)$ but not with $\mathrm{FEV}_{1} \%$ predicted. Capsaicin sensitivity was not related to the presence of infected sputum or to corticosteroid or bronchodilator use. Conclusions : Patients with bronchiectasis have a sensitive cough reflex which reflects the severity of cough symptoms. A measure of cough severity could be part of health assessment for patients with bronchiectasis.

B ronchiectasis is a chronic lung disorder characterised by irreversible dilatation of the bronchi associated with the chronic daily production of mucopurulent sputum. ${ }^{1}$ Many causes of bronchiectasis are recognised including cystic fibrosis, immunodeficiency, primary ciliary dyskinesia, chronic aspiration, rheumatic conditions, and inflammatory bowel disease. A chronic productive cough is usually the predominant symptom of the disease. Patients suffering from a chronic cough due to a wide variety of causes usually have an increased cough sensitivity to inhaled capsaicin, as has been shown in patients with asthma, chronic obstructive pulmonary disease (COPD), ${ }^{2}$ pulmonary fibrosis, ${ }^{3}$ and chronic idiopathic cough. ${ }^{4}$

The aim of this study was to determine capsaicin cough sensitivity in patients with bronchiectasis compared with healthy controls. Although cough in bronchiectasis is attributed to the need to clear excessive airway secretions and mucus, it is possible that there is also an increase in cough sensitivity that could contribute to the cough response in this condition. Such a possibility is supported by the presence of neutrophilic inflammation and epithelial damage that is observed in the airways of patients with bronchiectasis. ${ }^{5}$ We also investigated the relationship between capsaicin cough sensitivity and spirometry, radiological extent of the disease, corticosteroid and bronchodilator use, the presence of infected sputum, and cough symptom severity.

\section{METHODS}

\section{Study subjects}

Twenty two patients with bronchiectasis recruited from the Royal Brompton Hospital clinics and 20 healthy non-smoking controls matched for age and sex were studied during a period of stability defined by no changes in symptoms for 4 weeks and no use of antibiotics for 12 weeks. Patients whose bronchiectasis was associated with primary ciliary dyskinesia and allergic bronchopulmonary aspergillosis were excluded. None of the patients had a smoking history and all had a negative histamine challenge to exclude the diagnosis of asthma. All patients underwent capsaicin cough challenge, vitalographic measurements, and symptom and quality of life questionnaires on the same day.

The study was approved by the ethics committee of the Royal Brompton and Harefield Trust and informed consent was obtained from each patient.

\section{Measurements}

Capsaicin cough challenge was performed in all subjects by administration of incrementally doubling concentrations of capsaicin $(0.98-500 \mu \mathrm{M})$ inhaled from a nebuliser connected to a breath activated dosimeter (PK Morgan Ltd, Gillingham, UK). Two ml of solution were placed in the nebuliser that was driven at a pressure of $151 \mathrm{kPa}$ for a period of 1 second. Each puff delivered was $20 \mu \mathrm{l}$, with a particle size of $4 \mu \mathrm{m}$ mass median aerodynamic diameter. Each dose was administered at 1 minute intervals. Patients were instructed to inspire from functional residual capacity to total lung capacity at a flow rate to trigger the dosimeter. They were asked to cough freely and the number of coughs induced during the 1 minute period after capsaicin administration was counted, although most of the coughs occurred within 10-15 seconds of inhalation. The end point was the concentration of capsaicin required to induce 5 coughs (C5). Forced expiratory volume in one second $\left(\mathrm{FEV}_{1}\right)$ was measured in all subjects using a dry wedge spirometer (Vitalograph, Buckingham, UK).

An assessment of the impact of chronic coughing on quality of life in patients with bronchiectasis was made using the Leicester Cough Questionnaire (LCQ). ${ }^{6}$ This consisted of 19 cough related questions. A minimum total score of 19 indicates maximal impact on quality of life whereas a maximum total score of 133 indicates no impact on quality of life.

Severity of cough symptoms was also assessed using a previously described cough symptom score. ${ }^{7}$ Severity of

Abbreviations: $\mathrm{C} 5$, concentration of capsaicin required to induce 5 coughs; $\mathrm{FEV}_{1}$, forced expiratory volume in 1 second; $L C Q$, Leicester Cough Questionnaire 
daytime and night time cough symptoms were scored by the patients according to the following scales:

- During the day: $0=$ no cough; 1 = cough for one short period; $2=$ cough for two or more short periods; $3=$ frequent coughing, not interfering with usual daytime activities; $4=$ frequent coughing, interfering with usual daytime activities; $5=$ distressing coughs for most of the day.

- During the night: $0=$ no cough; $1=$ cough on waking in the morning only; 2 = wake once or early due to cough; $3=$ frequent waking due to coughs; $4=$ frequent coughs most of the night; $5=$ distressing coughs preventing any sleep.

Daytime and night time scores were added together to give the total cough symptom score with a maximum possible score of 10 .

High resolution computed tomographic (HRCT) scanning of the lungs was performed within 2 months of the capsaicin cough challenge. Radiological assessment of the severity of bronchiectasis was performed using a modification described by Bhalla et al. ${ }^{8}$ One observer (DHC), a radiologist, scored HRCT images of each of the six lobes (the lingula was regarded as a separate lobe) according to the severity of bronchial dilatation, bronchial wall thickening, and mucus plugging. Bronchial dilatation was scored relative to the diameter of adjacent pulmonary arteries: $0=$ normal; $1=$ $100-150 \%$ of arterial diameter; $2=150-200 \%$ arterial diameter; $3=>200 \%$ arterial diameter. Bronchial wall thickening was also scored relative to adjacent pulmonary arteries: $0=$ normal; $1=<50 \%$ arterial diameter; $2=50-$ $100 \%$ arterial diameter; $3=>100 \%$ arterial diameter. Mucus plugging was scored as follows: $0=$ absent; $1=$ present; 2 $=$ moderate; $3=$ marked.

Use of inhaled corticosteroids and bronchodilators by the patients was recorded and samples of sputum were sent for bacteriological assessment when the patients were recruited into the study. In case sputum samples were not provided on the day (as in six subjects), the patient was asked to provide a sputum specimen within the week. None of the patients were on oral corticosteroids.

\section{Analysis of data}

All statistical analyses were performed using GraphPad Prism Version 3.03. Unpaired $t$ tests were used to analyse differences in quantitative data (age, C5, FEV 1 ) between groups and the $\chi^{2}$ test was used to analyse dichotomous data. All correlation coefficients were calculated using the Pearson's test. A p value of $<0.05$ was considered statistically significant. Dates are expressed as mean (SE).

\section{RESULTS}

Baseline demographic data are shown in table 1. The groups were matched for age and sex. Patients with bronchiectasis had a significantly lower mean $\mathrm{FEV}_{1}$ (\% predicted) than controls (82.9 (6.3) v 99.9 (0.9); $\mathrm{p}<0.02)$. Thirteen $(59 \%)$ of

Table 1 Characteristics of patients with bronchiectasis and healthy controls

\begin{tabular}{lll}
\hline & $\begin{array}{l}\text { Bronchiectasis } \\
\text { (n= 22) }\end{array}$ & $\begin{array}{l}\text { Controls } \\
\text { (n= 20) }\end{array}$ \\
\hline Mean (SD) age (years) & $59(3)$ & $49(3)$ \\
Sex (n (\%) female) & $18(82 \%)$ & $16(80 \%)$ \\
Mean (SD) FEV $1 \%$ predicted) & $82.9(6.3)$ & $99.9(1.9)$ \\
Inhaled corticosteroid use & $13(59 \%)$ & 0 \\
Inhaled bronchodilator use & $13(59 \%)$ & 0 \\
\hline SD, standard deviation. & & \\
\hline
\end{tabular}

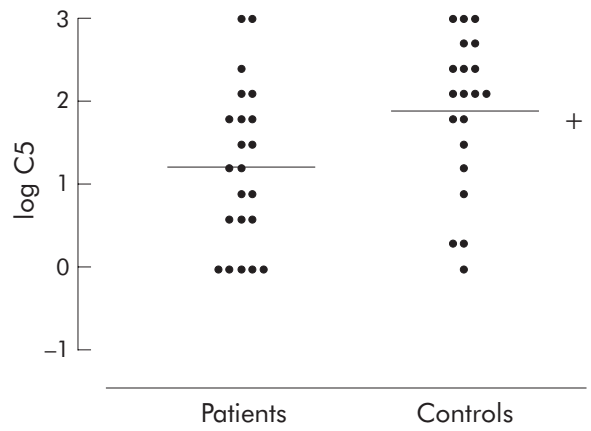

Figure 1 Comparison of capsaicin sensitivity $\left(\log _{10} \mathrm{C} 5\right)$ between patients with bronchiectasis and controls. Horizontal bars indicate mean. $\mathrm{tp}<0.03$ compared with patients with bronchiectasis.

the bronchiectatic patients were taking regular inhaled steroids and 13 (59\%) were taking inhaled bronchodilators. Of the 22 patients with bronchiectasis, 10 had culture positive sputum with the following organisms: Haemophilus influenzae (HI), Pseudomonas aeruginosa (PA), Streptococcus pneumoniae (SP), and Moraxella catarrhalis (MC). One patient had three organisms growing in the sputum $(\mathrm{HI}+\mathrm{MC}+\mathrm{SP})$; five patients had a combination of two organisms (two with $\mathrm{HI}+\mathrm{PA}$, two with $\mathrm{HI}+\mathrm{MC}$, and one with $\mathrm{HI}+\mathrm{SP}$ ).

Capsaicin data were normalised using logarithms of C5 values. Patients with bronchiectasis had a significantly lower mean $\log$ C5 than controls (1.22 (0.20) v 1.89 (0.21); $<<0.03$; fig 1), indicating heightened cough sensitivity. In nine of the 22 patients measurement of capsaicin cough sensitivity was repeated 1-2 years later. Analysis of these nine responses for repeatability according to the Bland and Altman method shows that the mean difference between the two log C5 measurements was 0.02 , and the limits of agreement within which $95 \%$ of the repeat measurements are estimated to lie were -0.98 and 1.38 .

The mean LCQ and cough symptom scores in the patients were $97(6)$ and $2.3(0.3)$, respectively. There was a strong negative correlation between the LCQ score and the cough symptom score $(r=-0.9 ; \mathrm{p}<0.0001 ;$ fig 2$)$. The mean CT scan score for these patients was 14.3 (1.2).

Capsaicin sensitivity $\left(\log _{10} \mathrm{C} 5\right)$ in the bronchiectatic patients was positively correlated with $\mathrm{FEV}_{\mathrm{l}}$ in litres $(r=0.58 ; \mathrm{p}=0.005 ;$ fig $3 \mathrm{~A})$ and with the LCQ score $\left(r=0.64 ; \mathrm{p}=0.005\right.$; fig 3B). However, $\mathrm{FEV}_{1}$ expressed as $\%$ predicted did not correlate with capsaicin sensitivity or with LCQ scores. There was a negative correlation between log C5 and the total simple cough score $(r=-0.58 ; \mathrm{p}=0.004$; fig 3C). There was no correlation between $\log \mathrm{C} 5$ and the

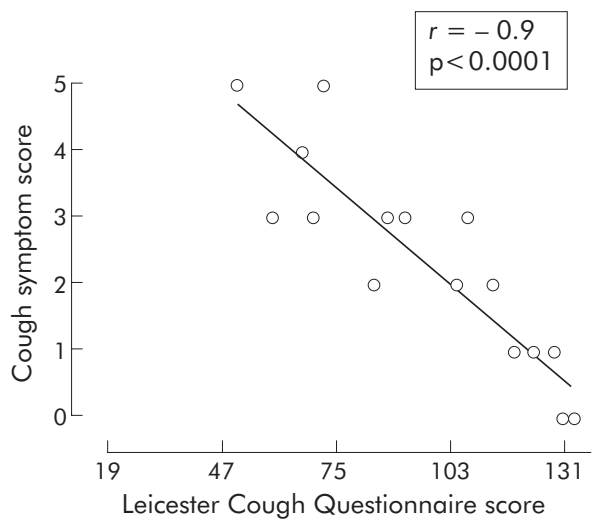

Figure 2 Relationship between Leicester Cough Questionnaire scores and cough symptom score in patients with bronchiectasis. 

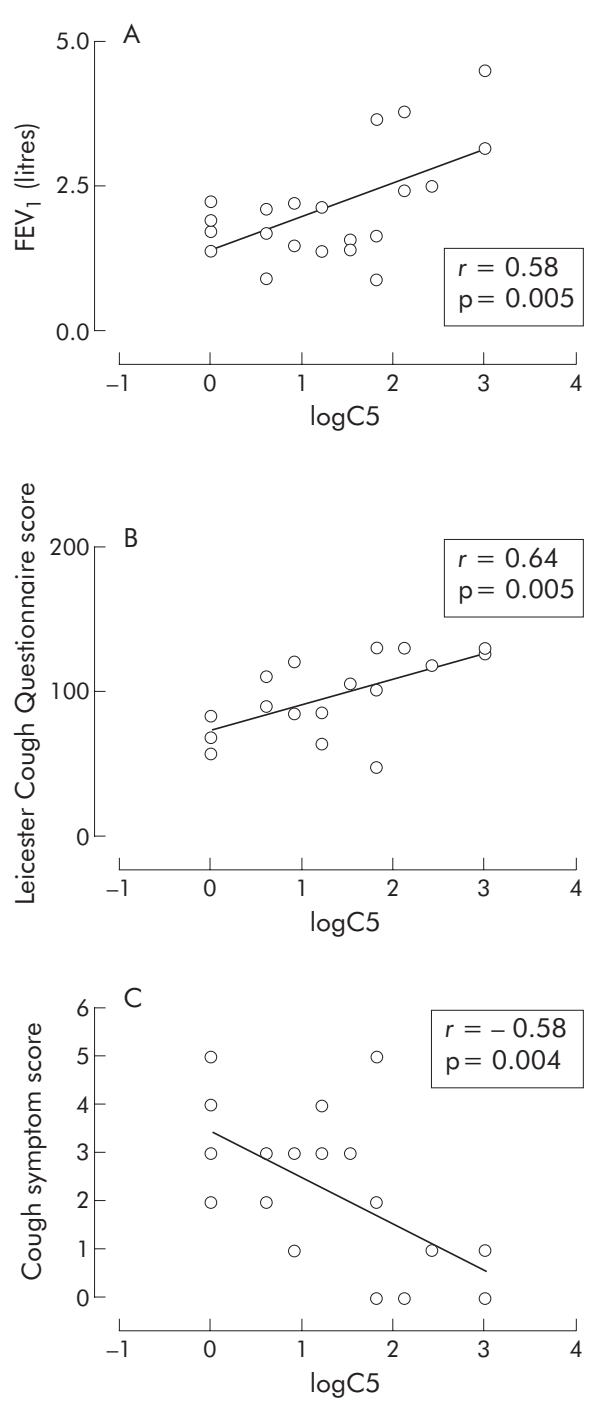

Figure 3 Relationship between capsaicin sensitivity $(\log C 5)$ and (A) $\mathrm{FEV}_{1}$, (B) Leicester Cough Questionnaire (LCQ) score, and (C) cough symptom score in patients with bronchiectasis.

radiological assessment (bronchial dilatation, bronchial wall thickening, mucus plugging, and air trapping). The presence of infected sputum and the organism grown from the sputum did not correlate with capsaicin sensitivity. Finally, the dose and use of bronchodilators or of inhaled corticosteroids did not relate to capsaicin sensitivity.

\section{DISCUSSION}

Bronchiectasis is primarily the result of airway injury and remodelling secondary to chronic inflammation and recurrent airway infections characterised by chronic cough and sputum production. We found a significant increase in cough reflex sensitivity, as measured with inhaled capsaicin, of the order of fourfold in a cohort of subjects with moderate to severe bronchiectasis. This degree of capsaicin cough sensitivity was correlated with $\mathrm{FEV}_{1}$, the severity of cough symptoms, and with the impairment of quality of life as measured by the LCQ. These data indicate that the degree of capsaicin sensitivity may be a good clinical marker to complement evaluation of the severity of bronchiectasis.

The capsaicin cough sensitivity of seven patients with bronchiectasis grouped among patients with a chronic productive cough has previously been reported. As a whole, this group had a similar capsaicin cough sensitivity to the control subjects who did not cough, but when the seven bronchiectatic patients were compared with the control group they were found to have a smaller capsaicin C5 value. ${ }^{9}$ The severity of the bronchiectatic disease was not reported, but they did have infected sputum. In our study we did not link the presence of infected sputum at the time of capsaicin measurement to the degree of capsaicin sensitivity, although it is likely that our study may have lacked the power to show such a relationship. We did not note the purulence of the sputum at the time, and our definition of infected sputum was the presence of significant bacterial growth from the sputum collected. It would be interesting to determine whether the purulence was related to capsaicin cough sensitivity. This larger study of patients with extensive bronchiectasis indicates that patients with this chronic pulmonary condition, most often presenting with chronic cough, have capsaicin hypersensitivity similar to other chronic lung diseases such as COPD, asthma, and pulmonary fibrosis. ${ }^{23}$

The factors involved in cough induction in bronchiectasis include the continuous presence of sputum and airway secretions, often containing bacteria, as in the majority of our patients. The mucus stasis is also compounded by damage to mucociliary clearance which represents damage to the epithelium. $^{10}$

We did not include patients with primary ciliary dyskinesia in our cohort because the cause of the bronchiectasis in this condition is primarily due to ciliary dysfunction which is different from abnormalities usually observed in the patients with "idiopathic" bronchiectasis that we studied. In addition, the occurrence of concomitant diseases such as rhinosinusitis or gastro-oesophageal reflux may contribute to or exacerbate cough. We have now shown that one additional factor that favours cough in bronchiectasis is the enhanced cough reflex. Underlying this could be the presence of chronic neutrophilic inflammation and damage that could lead to cough sensitisation. In bronchoalveolar lavage fluid obtained from patients with stable bronchiectasis, there are neutrophils and neutrophil products such as neutrophil elastase and myeloperoxidase and the proinflammatory cytokines tumour necrosis factor $\alpha$, interleukin (IL)-6 and IL-8. ${ }^{11}$ Release of inflammatory prostaglandins and bradykinin could lead to sensitisation of the cough reflex. In addition, the presence of bacterial infection is usually associated with more severe inflammation. ${ }^{12}$ Apart from the presence of mucus, an enhanced cough reflex may be related to neuroplasticity of both the central and peripheral cough pathways, such as overexpression of substance $\mathrm{P}$ in the brain stem nuclei. ${ }^{13}$ In the periphery there may be changes in the cough receptor caused by the neutrophilic inflammation such as an overexpression of transient receptor potential vanniloid-1 (TRPV1), the receptor for capsaicin. Aspects of neuroplasticity of the cough afferent pathways are also likely to contribute to the heightened reflex associated with chronic cough. ${ }^{14}$

The severity of cough has not been well studied in bronchiectasis. Although the St George's Respiratory Questionnaire (SGRQ) contains some specific questions on cough, the extent to which the presence of a chronic cough contributes to the overall impact on quality of life has not been reported. ${ }^{15}$ SGRQ scores have been shown to correlate well with measures of wheeze or breathlessness in bronchiectasis. It may be important to add more specific questions relating to the severity of cough since this seems to contribute significantly to the overall impairment of quality of life in patients with bronchiectasis. The LCQ, on the other hand, is a newly devised health status measure which specifically focuses on cough. Patients with bronchiectasis scored a maximum score of 133 (range 50-133), indicating moderate impairment of quality of life resulting from chronic cough. In addition, this score correlated closely with a simpler cough symptom score that only recorded the degree of coughing, 
and also with the precise single measure of capsaicin cough sensitivity. Capsaicin cough sensitivity may contribute to the clinical severity of bronchiectasis.

Cough can be a nuisance in bronchiectasis and contributes to deterioration in quality of life. However, cough should not be totally suppressed in bronchiectasis since it provides the only effective mechanism whereby excessive secretions are removed from the large airways.

\section{ACKNOWLEDGEMENTS}

The authors thank Drs Birring and Pavord of Glenfield Hospital, Leicester for the use of the Leicester Cough Questionnaire in our patients and Justine Arbery for help with the recruitment of patients.

\section{Authors' affiliations}

A Torrego, R A Haque, L T Nguyen, M Hew, D H Carr, R Wilson, K F Chung, National Heart and Lung Institute, Imperial College and Royal Brompton Hospital, London, UK

Funding: none.

Competing interests: none.

\section{REFERENCES}

1 Barker AF. Bronchiectasis. N Engl J Med 2002;346:1383-93.

2 Doherty MJ, Mister R, Pearson MG, et al. Capsaicin responsiveness and cough in asthma and chronic obstructive pulmonary disease. Thorax 2000;55:643-9.
3 Hope-Gill BD, Hilldrup S, Davies C, et al. A study of the cough reflex in idiopathic pulmonary fibrosis. Am J Respir Crit Care Med 2003;168:995-1002

4 Haque RA, Usmani OS, Barnes PJ. Chronic idiopathic cough: a discrete clinical entity? Chest 2005;127:1710-3.

5 Venaille TJ, Mendis AH, Phillips MJ, et al. Role of neutrophils in mediating human epithelial cell detachment from native basement membrane. J Allergy Clin Immunol 1995:95:597-606.

6 Birring SS, Prudon B, Carr AJ, et al. Development of a symptom specific health status measure for patients with chronic cough: Leicester Cough Questionnaire (LCQ). Thorax 2003;58:339-43.

7 Hsu JY, Stone RA, Logan-Sinclair RB, et al. Coughing frequency in patients with persistent cough: assessment using a 24 hour ambulatory recorder. Eur Respir J 1994;7:1246-53.

8 Bhalla M, Turcios N, Aponte V, et al. Cystic fibrosis: scoring system with thinsection CT. Radiology 1991;179:783-8.

9 Choudry NB, Fuller RW. Sensitivity of the cough reflex in patients with chronic cough. Eur Respir J 1992;5:296-300.

10 Del Donno M, Bittesnich D, Chetta A, et al. The effect of inflammation on mucociliary clearance in asthma: an overview. Chest 2000;1 18:1142-9.

11 Angrill J, Agusti C, De Celis R, et al. Bronchial inflammation and colonization in patients with clinically stable bronchiectasis. Am J Respir Crit Care Med 2001; 164:1628-32.

12 Wilson R. Bacteria, antibiotics and COPD. Eur Respir J 2001;17:995-1007.

13 Mutoh T, Bonham AC, Joan JP. Substance P in the nucleus of the solitary tract augments bronchopulmonary C fiber reflex output. Am J Physiol Regul Integr Comp Physiol 2000;279:1215-23.

14 Undem BJ, Carr MJ, Kollarik M. Physiology and plasticity of putative cough fibres in the guinea pig. Pulm Pharmacol Ther 2002;15:193-8.

15 Wilson CB, Jones PW, O'Leary CJ, et al. Validation of the St George's questionnaire in bronchiectasis. Am J Respir Crit Care Med 1997; 156:536-41. 